\title{
Regulation of 2D DNA Nanostructures by the Coupling of Tile Cur- vatures and Arm Twists
}

\author{
Chuan Jiang ${ }^{1, \circ}$, Biao Lu ${ }^{1, \circ}$, Wei Zhang ${ }^{1}$, Yoel P. Ohayon ${ }^{2}$, Caihong Ni ${ }^{1}$, Feiyang Feng ${ }^{1}$, Nadrian C. \\ Seeman $^{2, *}$, Shou-Jun Xiao ${ }^{1, *}$ \\ ${ }^{1}$ State Key Laboratory of Coordination Chemistry, School of Chemistry and Chemical Engineering, Nanjing University, \\ Nanjing 210023, China. \\ ${ }^{2}$ Department of Chemistry, New York University, New York, NY 10003, USA \\ *Corresponding authors, E-mails: ned.seeman@nyu.edu, sjxiao@nju.edu.cn
}

\begin{abstract}
DNA overwinding and underwinding between adjacent Holliday junctions have been applied in DNA origami constructs to design both left-handed and right-handed nanostructures. For a variety of DNA tubes assembled from small tiles, only an abstract concept of the intrinsic tile curvature was previously used to explain their formation. Details regarding the quantitative and structural descriptions of the intrinsic tile curvature and its evolution in DNA tubes by coupling with arm twists have been lacking. In this work, we designed three types of tile cores from a circular 128 nucleotide scaffold by longitudinal weaving (LW), bridging longitudinal weaving (bLW), and transverse weaving (TW) and assembled their 2D planar or tubular nanostructures via inter-tile arms with a distance of an odd or even number of DNA half-turns. The biotin/streptavidin (SA) labeling technique was applied to define the tube configuration with addressable inside and outside surfaces and thus their component tile conformation with addressable concave and convex curvatures. Both chiral tubes possessing left-handed and right-handed curvatures could be generated by finely tuning $\mathrm{p}$ and $\mathrm{q}$ in $\mathrm{bLW}-\mathrm{E}_{\mathrm{p} / \mathrm{q}}$ designs ( $\mathrm{bLW}$ tile cores joined together by inter-tile arms of even number of half-turns with the arm length of $p$ base pairs (bp) and the sticky end length of q nucleotides (nt)). We were able to assign the chiral indices (n,m) to each specific tube from the high-resolution AFM images, and thus estimated the tile curvature angle with a regular polygon model that approximates each tube's transverse section. We attribute the curvature evolution of bLW- $\mathrm{E}_{\mathrm{p} / \mathrm{q}}$ tubes composed of the same tile core to the coupling of the intrinsic tile curvature and different arm twists. A better understanding of integrated actions of different types of twisting forces on DNA tubes will be much more helpful in engineering DNA nanostructures in the future.
\end{abstract}

\section{INTRODUCTION}

For the past 40 years, the field of DNA nanotechnology has relied on the programmability of Watson-Crick base pairs (bp) to construct many versatile DNA nanostructures and nanodevices. ${ }^{1-8}$ The organization of 1D, 2D, and 3D DNA nanostructures can be divided into two hierarchically coherent architectures, namely weaving and tiling. Weaving relies on winding soft, thread-like, single-stranded DNAs into double-stranded helixes (hereafter "helix" will represent "double-stranded helix") and crossovers mainly three- and fourbranched junctions, forming small tiles of nanoscale dimensions, ${ }^{9-13}$ larger origami tiles of tens of nanometers, ${ }^{3,14}$ or single-stranded tile and brick lattices of (sub)micrometers. ${ }^{15-17}$ Each tile can be categorized into two sets: a tile core surrounded by the scaffold strand including boundary junctions, and outer overhangs carrying the helix stems and joints of sticky ends. Tiling, a term originated from the construction architecture, refers here to the assembly of DNA tiles into either finite or infinite nanostructures by joining tiles with inter-tile arms via sticky end cohesion. In our current work, we term the joining with the arm length of an even number of half-turns as E-tiling and that of an odd number of half-turns as O-tiling. In tiles, the intrinsic tile curvature is originated from weaving, whereas the arm twist is generated from tiling. Coupling of both will finely tune the 2D DNA nanostructures into different morphologies and 
chirality. From 1D to 3D periodic DNA nanostructures, Bravais lattices can be abstracted, with tile cores as lattice points, joining arms as bonds, and the smallest repeating structures as unit cells. Thus, the 2D planar and tubular lattices in this work will be described through crystal terms and analyzed using crystal theory.

\section{Design strategies}

A small DNA tile, such as a double crossover $(\mathrm{DX})^{9}$ tile or a multi-arm junction tile ${ }^{18-19}$ composed of several oligonucleotides, is often woven via a few four-branched Holliday junction (HJ) crossovers to only one specific weaving pattern because of the space limits of its weaving architecture. Recently, small circular DNA molecules have been used as scaffolds to build cDAO tiles (coupled double crossovers composed of two antiparallel helixes with a distance of an odd number of half turns between adjacent crossovers), 1D nanowires, 2D nanoribbons as well as nanotubes. ${ }^{20-21}$ The cDAO tile assembled from a circular 64 nt strand (abbreviated as c64nt) is composed of two DAO motifs coupled in series. However, with a circular 84 nt scaffold (c84nt), the center-crossover motif HJc84nt failed to form two DAE (double crossovers composed of two antiparallel helixes with a distance of an even number of half turns between the crossovers) tiles coupled in series, and thus it was unable to assemble 2D lattices. The reason was that the incoming and outgoing nucleosides of each helper strand at its corresponding pole forms a nick, and the nick was located inside and restrained by the c84nt scaffold loop. Such a failure drove us to think about weaving a circular DNA tile with two DAE motifs coupled in parallel. Increasing the circular scaffold to a large size of circular $128 \mathrm{nt}$ (c128nt), twice of c64nt, we folded it into two shapes of outlined letters of $\mathrm{H}$ and $\mathrm{X}$ (or called $\mathrm{H}$-shape and X-shape), and obtained three weaving architectures to build the following tiles and 2D nanostructures: 1) traditional longitudinal weave (LW) along the H-shaped scaffold to generate LW tile cores and LW-E $/ \mathrm{p} / \mathrm{LW}$ $\mathrm{O}_{\mathrm{p} / \mathrm{q}}\left(\mathrm{E}_{\mathrm{p} / \mathrm{q}}\right.$ means an arm length of an even number of half-turns composed of $\mathrm{p}$ base pairs $(\mathrm{bp})$ and a sticky end length of $\mathrm{q}$ nucleotides (nt), and $\mathrm{O}_{\mathrm{p} / \mathrm{q}}$ means an arm length of an odd number of half-turns composed of $\mathrm{p}$ bp and a sticky end length of $\mathrm{q}$ nt) nanostructures, 2) bridging longitudinal weave (bLW) along the X-shaped scaffold by inserting a $10 \mathrm{bp}$ helix at the center to yield bLW tile cores and $\mathrm{bLW}-\mathrm{E}_{\mathrm{p} / \mathrm{q}} / \mathrm{bLW}-\mathrm{O}_{\mathrm{p} / \mathrm{q}}$ nanostructures, 3) transverse weave (TW) of the H-shaped scaffold to produce TW tile cores and TW$\mathrm{E}_{\mathrm{p} / \mathrm{q}} / \mathrm{TW}-\mathrm{O}_{\mathrm{p} / \mathrm{q}}$ nanostructures.

\section{Definition and estimation of tile curvature}

Because of the worm-like or spring-like structures of DNA molecules, a well-known phenomenon in DNA nanotechnology is that DNA nanotubes and planar ribbons are often observed when E-tiling and O-tiling are applied, respectively; this has been explained to be caused by the intrinsic tile curvature. ${ }^{18}$ Following the natural B-DNA winding phase, E-tiling requires that all tile faces to be aligned identically, and O-tiling requires that adjacently joined tile faces to be aligned alternately. By assuming that the arms in both E-tiling and O-tiling tactics are straight and do not generate any torque, the intrinsic tile curvatures will be accumulated by E-tiling to generate tubes, whereas they will be cancelled out by O-tiling both locally and globally to produce planar ribbons. Such qualitative explanations regarding tile curvature have been proven to be successful in most cases. However, details and quantitative descriptions about its contents and scopes have been lacking, such as the tile conformation (which tile face is concave or convex?), the curvature angle of the tile with a defined orientation, and the curvature transition and transformation (does a tile curvature evolve gradually or even flip-over) with changing arm lengths and/or sticky end lengths. The absence of such data in the design toolbox of DNA nanotechnology has been constituting a hurdle to realize precise and diverse DNA nanostructures efficiently.

A well-established strategy to design curled DNA nanostructures with addressable surfaces is the periodic insertion or deletion of

base pairs in or from natural B-DNA segments between adjacent HJs, resulting in net torsional strains on the segments and thus forming the right-hand or left-hand twisted beams and tubes. ${ }^{22-24}$ Such curving effects with overwinding and underwinding strategies have been applied in DNA origami and single-stranded tile/brick nanostructures via the accumulation of periodically biased torques to yield left-handed and right-handed constructs, respectively. ${ }^{25-29}$ In the two systems of DNA origami and single-stranded tiles, DNA weaving and tiling architectures are integrated together, in which the DNA tile cores and E/O-tiling arms cannot be distinguished 
clearly. While for a large number of DNA tubes assembled from small tiles via E-tiling, ${ }^{30-34}$ only a very few reports briefly mentioned about their addressable inside and outside surfaces, as well as discussed about the tube perimeters and morphologies. ${ }^{35-36}$ Many questions regarding the intrinsic tile curvature and its evolution with changing arm and sticky end lengths still remain open.

In DNA tubular structures of DAE-E tubes, ${ }^{35}$ six helix bundle tubes, ${ }^{37-38}$ and single-stranded tile tubes, ${ }^{16,39}$ the universal polygon model has been applied to explain the curving of tile arrays to tubes. Such polygon models are based on the B-DNA winding criteria including the major/minor grooving effect at crossovers by presupposing that all helixes are rigid, straight, tangent between adjacent helixes, and parallel to the tube axis. However, a precise physical description of the tile curvature and its cooperation with armtwisting forces have not yet been illustrated clearly in the polygon models mentioned above.

In this work, in order to quantify the tile curvature, we first relied on high-resolution AFM images of each type of 2D lattices with a lateral resolution at around $2 \mathrm{~nm}$ to abstract its $2 \mathrm{D}$ Bravais lattice of centered rectangle and precisely measured the unit cell parameters (Section S2, S3 and S4 of SI). It is obvious that only the E-tiling tubes are adequate for the curvature analysis. Secondly, we described each specific tube with the chiral indices $(n, m)^{33,40}$ based on its high-resolution AFM image. The correspondence of the tube perimeters from theoretical calculations based on $(n, m)$ and lattice linear and angular constants to experimental measurements confirmed the correct assignment of $(n, m)$. Thirdly, we projected the tube unit cell possessing the chiral indices (n,m) (in this work, we limited our analysis to two types of stable tubes, $n=2 m$ and $n=m$ ) onto its transverse section and approximate the projection to a regular polygon model. The exterior angle of the regular polygon is assigned as the tile curvature angle. We define this semi-quantified curvature as the global tile curvature, which integrates the intrinsic tile curvature and the arm twist together. We define the intrinsic tile curvature ideally as the curvature of a free tile, which depends on its weaving architecture. However, its measurement is beyond our current capabilities. In practice, we could assign the global tile curvature defined from an E-tiling tube with an arm length of 21 bp and a sticky end length of $5 \mathrm{nt}$ (specified as $\mathrm{E}_{21 / 5}$ ) as the intrinsic tile curvature, which is based on the postulation that the $\mathrm{E}_{21 / 5}$ arm is straight and does not yield any additional torque. ${ }^{22}$ Under such a postulation, we also considered the intrinsic tile curvature as the intrinsic curvature of the tile core.

We relied on combination of both biotin/SA labeling and high-resolution AFM imaging techniques to define the configurations of the E-tiling tubes and thus the conformations of the DNA tiles, primarily for the stable and minimally distorted tubes wound according to the natural B-DNA helixes. We represent the stereo-conformation or chirality of a tile with the right-hand or left-hand grip rule. To demonstrate, with the thumb points towards the inside surface of the tube or the concave face of the tile, the rotation direction of c128nt from 5' to 3' can be followed by the right-hand or left-hand grip. For concise presentation, we use $l$ - or $r$ - as the prefix of the chiral indices $(\mathrm{n}, \mathrm{m})$ of a specific tube as $l-(\mathrm{n}, \mathrm{m})$ or $r-(\mathrm{n}, \mathrm{m})$ to represent its left-handed or right-handed curvature. Similarly, we separated and identified the two opposite faces of a tile using either the $l$-face or the $r$-face. To add a note here, the prefix of $l$ - or $r$ in front of $(\mathrm{n}, \mathrm{m})$ indicates a clear geometry of the tube, whereas $l$ - or $r$ - in front of "face"only indicates one of the two opposite faces of a tile exclusively.

\section{RESULTS AND DISSCUSSION}

\section{Synthesis of c128nt and tile stability}

The circular c128nt was synthesized using two linear, phosphorylated 64 nt oligonucleotides and their two corresponding splints by T4 ligation. The c128nt was purified through denaturing polyacrylamide gel electrophoresis (PAGE) with a yield of 40 60\% (Section S1.2 and Figure S1). We used the native PAGE to test the stability and yield of three tile cores of LW, bLW, and TW (Section S1.4 and Figure S2). For each core, a single band confirms its stability. The yield of about $80 \%$ for each core was analyzed with the software "imageJ" of the gel band intensity in each lane.

\section{A unified 2D Bravais lattice of centered rectangle}


From both theoretical designs and experimental results of AFM images, we applied a unified 2D Bravais lattice of centered rectangle to describe all the 2D DNA lattices originated from c128nt-derived tiles. For example, in the brick assembly model of Figure 1A(a), a compound unit cell of centered rectangle containing two tiles is defined by the mutually orthogonal basis vectors $\boldsymbol{a}$ and $\boldsymbol{b}$ with unit lengths $a$ and $b$, respectively, and a primitive cell of rhombus containing one tile is superimposed by the primitive vectors $c_{1}$ and $c_{2}$ with unit length $c$ and inter-angle $\varphi$. By achieving a lateral resolution of about $2 \mathrm{~nm}$ in AFM images, we were able to measure the unit cell linear and angular constants of each distinct 2D lattice. The measured lattice constants were in line with the simple theoretical estimations of our designs well (theoretical descriptions refer to Section S2 and S3, data refer to Table 1 and Table S1).

\section{D arrays of longitudinal weaving (LW) tiles}

In Figure 1, both designs of LW- $\mathrm{E}_{21 / 5}$ and LW-O $\mathrm{O}_{26 / 4}$ are shown with their brick assembly models (A(a) with face-identical packing and B(a) with face-alternating packing), helix tile models (A(b) and B(b)), and zoomed-out (A(c) and B(c)) and zoomed-in (A(d) and B(d)) AFM images (more images are shown in Figures S12 and S13). In the AFM images of the LW- $\mathrm{E}_{21 / 5}$ and LW-O $26 / 4$ arrays, we found a majority of planar ribbons and a minority of wide tubes. In contrast with the general rule that the E-tiling results in tubes, LW- $E_{21 / 5}$ assemblies represented the majority of planar ribbons and lengthy monolayer fibers (Figure S12), meaning that the longitudinal weaving of H-shaped c128nt resulted in tightly folding, crowding, rigid, and planar LW cores with minimal curvature, similar to our previously reported cDAO-c64nt system. ${ }^{20}$ According to the traditionally faceted crystal growth theory by means of bond directions, lattice edges along $\boldsymbol{c}_{1}$ and $\boldsymbol{c}_{2}$, so-called flat faces, would appear thermodynamically, whereas edges along $\boldsymbol{a}$ and $\boldsymbol{b}$ (the socalled stepped faces) would occur dynamically. In both LW-E $21 / 5$ and LW-O $\mathrm{O}_{26 / 4}$ designs, either $\boldsymbol{c}_{1}$ or $\boldsymbol{c}_{2}$ only had a small inter-angle (less than $20^{\circ}$ ) with $\boldsymbol{a}$, and the aspect ratio of $a / b$ would be larger than 1.5 , thus, favoring the dynamic growth direction for both designs along $\boldsymbol{a}$. The lengthy edges (stepped faces) of planar ribbons and the tube axis in their AFM images are usually along $\boldsymbol{a}$.

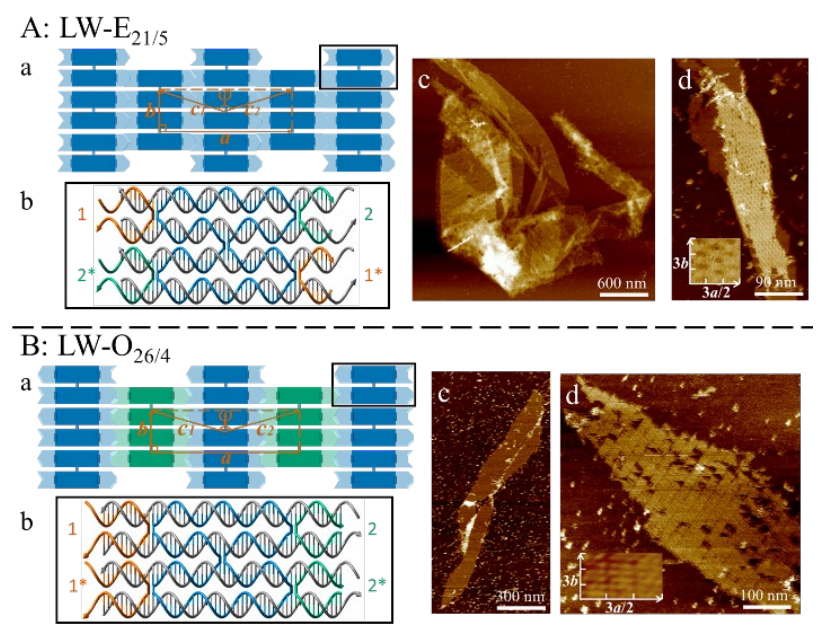

Figure 1. Longitudinal weaving and tiling systems of $\mathbf{L W}-\mathbf{E}_{21 / 5}$ and $\mathbf{L W}-\mathbf{O}_{26 / 4}$. (A) LW-E21/5: a) an assembly model for E-tiling with all the brick modules colored blue (the $l$-face), meaning having face-identical packing; b) a helix tile model; c and d) zoomed-out and zoomedin AFM images. (B) LW-O26/4: a) an assembly model with adjacent brick modules along the horizontal direction colored blue for the $l$-face and green for the $r$-face alternately, which indicates face-alternating packing; b) a helix tile model; c and d) zoomed-out and zoomed-in AFM images. In all the tile helix models of Figures $2 \mathrm{~A}(\mathrm{~b})$ and $2 \mathrm{~B}(\mathrm{~b})$, the blue strand c128nt acts as the central scaffold and rotates clockwise following the $5^{\prime} \rightarrow 3^{\prime}$ direction, thus, the $l$-face always faces up, and its opposite $r$-face always faces down; the same number pairs of $\left(1,1^{*}\right)$ or $\left(2,2^{*}\right)$ will cohere together to form arms whose length is defined as the inter-tile distance between adjacent HJs. In both the brick assembly models of Figures 1A(a) and 1B(a), the tile brick module is divided into two parts of deep-colored inner core and light-colored outer overhangs carrying double helix stems and sticky end joints, and decorated with several carved short bars representing HJs; a centered rectangle unit cell (defined by the basis vectors $a$ and $b$ ) and a plain rhombus unit cell (defined by the primitive vectors $c_{1}$ and $c_{2}$ ) with an inter-angle 
$\varphi$ are superimposed. In the zoomed-out AFM images, each inset shows the organization of 9 tiles in the Cartesian coordinates of $a$ and $b$, clearly illustrating the individual tile cores and their joining regions. The measured lattice constants are $a=34.6 \mathrm{~nm}, b=12.0 \mathrm{~nm}, c=18.3$ $\mathrm{nm}, \varphi=142^{\circ}$ for LW-E $21 / 5$; and $a=37.4 \mathrm{~nm}, b=12.0 \mathrm{~nm}, \mathrm{c}=21.1 \mathrm{~nm}, \varphi=149^{\circ}$ for $\mathrm{LW}-\mathrm{O}_{26 / 4}$.

Because we did not label any AFM imaging markers on LW-derived tiles, their densely woven 2D arrays (as well as TW-E $21 / 5$ and TW-O $\mathrm{O}_{26 / 4}$ assemblies which are described later) were primarily imaged in fluid as brighter patches of planar ribbons, handkerchieflike structures, or blurring tubes without any texture, as shown in Figures 1A(c), 1B(c), 3A(c), 3B(b), as well as in Figures S12, 7, S21, and S22. To obtain high-resolution images with nanoscale textures, we developed two imaging skills: 1) On a wide tube, the top monolayer sits flatways on a bottom monolayer and both layers are electrostatically repulsive. The nanoscale rhombus texture of the top layer was easily resolved with 1 to 3 scans, shown in Figure 1A(d) and magnified in the left bottom insert. In comparison with the brick assembly model of Figure 1A(a), each bright intersection point represents a LW core, each edge a 21 bp joining arm, and each dim pit a rhombus center torn a little bit apart by scanning. 2) For the LW-O $26 / 4$ monolayer array which was strongly adsorbed on the hard substrate of mica, its high-resolution image in Figure 1B(d) was achieved through repeated scans at least 4 times. However, repeated scans removed some tiles from the lattice patch and generated scattered empty pores. The hierarchical and distinctive imaging features in Figure 1B(d) was read out as follows: First, alternately bright tile arrays with a tile width corresponding to brick module columns, and dim, fine lines corresponding to sticky end cohesion columns ( $4 \mathrm{bp}$ or $0.32 \mathrm{~nm} \times 4=1.3 \mathrm{~nm}$ wide) of Figure 1B(a) were parallel to each other along $\boldsymbol{b}$, with a periodic distance of $a / 2$. Secondly, the magnification of three bright tile arrays in the insert of Figure 1B(d) shows that in the bright tile array each bright bar alternates with each dim line, in which the bright bar corresponds to either the upper or the lower part of the two helixes of a tile, and the dim line corresponds to the gap between two helixes. Occasionally, the central HJ connecting both upper and lower halves of a tile could also be imaged as a bright dot. We hypothesize that the strong adsorption of the DNA helixes on hard mica contributed to the higher lateral resolution in Figure 1B(d) than in Figure $1 \mathrm{~A}(\mathrm{~d})$ to the upper and lower halves of a tile resolved. The measured unit cell parameters of both LW-E $\mathrm{E}_{21 / 5}$ and LW$\mathrm{O}_{26 / 4}$ lattices are listed in the caption of Figure 1, which are in line with their theoretical estimations (Section S2 and Table S1). The high-resolution imaging details also confirm the correct Bravais lattice assignment of the centered rectangle.

\section{Chiral indices and curvature evolution of $b L W-E_{p / q}$ tubes}

The center-bridging strategies to build 2D bLW-derived nanostructures are demonstrated in Figure 2. The insertion of a flexible 10 bp helix instead of the central HJ releases more spatial room and provides more flexibility for the bLW-derived tiles to regulate themselves during assembly.

We show the O-tiling bLW-O $\mathrm{O}_{26 / 4}$ assemblies first. Figure 2A(a) shows an assembly model with face-alternating brick modules and Figure 2A(b) shows a helix tile model. Figure 2A(c) illustrates one of the widest tubes with a perimeter of $1.6 \mu \mathrm{m}$ (twice of the squashed double layer width). The zoomed-in image in Figure 2A(d) illustrates the tiles details. At each intersection point of the lattice, two tiny faint pores can be seen separated by the $10 \mathrm{bp}$ bridge. Thus, the geometry of the bLW core is defined as an X-shape with two pairs of opposite angles of around $60^{\circ}$ and $120^{\circ}$. The X-shaped geometry can be explained by the fact that the two three branch junctions at both sides could be kinked more easily than the two continuous helixes without any breaks at the top and at the bottom, as shown in the helix tile model of Figure $2 \mathrm{~A}(\mathrm{~b}) . \mathrm{bLW}-\mathrm{O}_{26 / 4}$ assemblies provide the dominant tubes with scattered perimeters (Figure S14).

In Figure 2B, we indicate three sets of chiral indices $(n, m)$ to specify DNA tubes. The chiral indices have been widely used in defining the carbon nanotube structures, ${ }^{41}$ but they have rarely been used in describing the DNA tube structures. ${ }^{33}$ On the hexagonal lattice $(\varphi$ $=120^{\circ}$ ) background of the $\mathrm{bLW}-\mathrm{E}_{\mathrm{p} / \mathrm{q}}$ assemblies with $l$-faces facing up, we drew the Bravais unit cells at the bottom left, and three sets of chiral indices, $(8,4)$ in purple shadow representing a typical bLW-E $\mathrm{E}_{21 / 5}$ tube unit cell, $(3,3)$ in green shadow representing the unique bLW-E $\mathrm{E}_{31 / 7}$ tube unit cell, and $(23,-23)$ in blue shadow representing a typical "giant" bLW-E $32 / 6$ tube unit cell. The chiral indices $(\mathrm{n}, \mathrm{m})$ are abstracted from $\mathrm{n} \boldsymbol{c}_{1}+\mathrm{m} \boldsymbol{c}_{2}$, which is the chiral (or perimeter) vector $\boldsymbol{C h}(\mathrm{n}, \mathrm{m})$ originated from the origin $\mathbf{O}$. For example, 
$(8,4)$ and $(3,3)$ are abstracted from $\boldsymbol{C}_{\boldsymbol{h}}(8,4)=8 \boldsymbol{c}_{\boldsymbol{1}}+4 \boldsymbol{c}_{2}$ and $\boldsymbol{C}_{\boldsymbol{h}}(3,3)=3 \boldsymbol{c}_{\boldsymbol{1}}+3 \boldsymbol{c}_{2}$, respectively. The tube unit cell of $(23,-23)$ is represented with repeating units at the top right, in which vectors of $23 c_{1}$ and $-23 c_{2}$ are omitted for illustration purposes. The unit cell parameters of $c$ and $\varphi$ were measured directly from the AFM images, thus, the chiral indices (n,m) could precisely define many tube unit cell parameters, including the perimeter (also diameter and radius), the helical angle of the inter-angle between $\boldsymbol{c}_{2}$ and $\boldsymbol{C h}(\mathrm{n}, \mathrm{m})$, and the axial periodicity $(\boldsymbol{T})$ (Section S3 and Figure S4), and also the global tile curvature being investigated in our study. ${ }^{40}$

Combining both the biotin/SA labeling and the AFM imaging techniques, we analyzed the E-tiling tubes of bLW-E21/5 in Figures $2 \mathrm{C}(\mathrm{a}-\mathrm{f}), \mathrm{bLW}-\mathrm{E}_{31 / 7}$ in Figures 2D(a-e), and bLW-E $\mathrm{E}_{32 / 6}$ in Figures 2E(a-e) in detail. The evolution of bLW-E $\mathrm{E}_{\mathrm{p} / \mathrm{q}}$ tubes in perimeters, the tube configurations, the tile conformations, and global tile curvatures by manipulating both the arm and sticky end lengths enabled us to gain a much deeper understanding of the tube formation mechanism.

For the bLW-E $E_{21 / 5}$ tubes, Figure $2 \mathrm{C}(\mathrm{a})$ shows the schematic brick model of the typical tube $(8,4)$ (top) and the helix model of its component tile (bottom) with a biotin label at overhang 2. Figure $2 \mathrm{C}(\mathrm{b})$ provides a zoomed-out AFM image containing many tubes (top) and a zoomed-in image of only one typical tube (bottom). Densely distributed tubes in the top panel suggest a high yield for the products. Figure $2 \mathrm{C}(\mathrm{c})$ shows an abundance of 73 tubes falling in 4 perimeter windows, in which tube perimeters were measured from more than 5 batches of assemblies (Section S5, Table S2, and block 1 of Figure S15). Obviously, the tubes are relatively homogeneous, falling within the perimeter window of 120.0 to $240.0 \mathrm{~nm}$. An obvious feature of the bLW-E21/5 tubes in all the zoomedin images (Figures $2 \mathrm{C}(\mathrm{b}, \mathrm{d})$, Figures S4-9 and S15) was that the two groups of $c_{1}$ and $c_{2}$-frames were not symmetric. One group of frames were aligned nearly parallel to the tube axis, and the other group curled around the tube axis with left-handed rolling.

Figure $2 \mathrm{C}(\mathrm{d})$ shows the $l$-face brick assembly model with each tile carrying a biotin/SA label (top), and the real zoomed-in image of the inside surface of a tube (bottom). The ideal match of the periodic structure of SA dots (each SA dot is around 5.0 to $6.0 \mathrm{~nm}$ in diameter $)^{39}$ overlying on the background lattice between the real image and the model refers that the intrinsic tile curvature follows the left-hand grip rule.

We describe here more experimental details for our reasoning process. First, the tubes must be in the open form of monolayers to image both of the background lattice structure and the overlying SA dots clearly, as shown in the bottom panel of Figure 2C(d) and in block 2-4 of Figure S15. DNA tubes are often reported to be open by repeated scanning of the same region. ${ }^{42-44}$ Herein, we report another experimental solution to prepare DNA tubes mostly either in the open form or in both the open and closed forms more conveniently by regulating the air-blowing strength. After $2 \sim 4$ min period of incubation of the sample drop on mica to let DNA tubes be adsorbed, we washed the sample specimen with water and blew the water drop away with air streams (Section S1.6 and Figure S3). The top layers of the tubes were either partly or completely removed with water together by surface tension interactions, whereas the bottom layers were strongly adsorbed and remained intact on the mica. Then, AFM scanning in either air or buffer was carried out separately. The completely or partly broken $b L W-E_{21 / 5}$ tubes were imaged mostly as monolayer strips, which were either locally straight or kinked at some sites. The monolayer strips exposing the tube inside surface towards us is a requirement to define the curvature direction. For purposes of being concise, we only show the results of biotin/SA labeling at the 5'-end of the outer helper strand (green strand) of overhang 2 for bLW-E $\mathrm{E}_{21 / 5}, \mathrm{bLW}-\mathrm{E}_{31 / 7}$, and bLW-E $\mathrm{E}_{32 / 6}$ in Figure 2 (additional AFM images of each design are shown in Figures S15, S19, and S20). The other biotin/SA labeling results at overhang 1 and/or 1* are also shown in Figures S15-20. The in-situ binding of the SAs on biotin-labeled lattices was carried out under a dilute SA solution according to previously reported protocols. ${ }^{18,39}$ Although the biotin-labeled nucleoside is located at the buried $r$-face in Figure $2 \mathrm{C}(\mathrm{d})$, high yield of binding of SAs(70 90 \%) was confirmed by AFM imaging because the empty space between the arms allowed biotins to stand up from the mica, exposed their functional groups to the outside, and bound the SAs easily. From the massive SA dots overlying on bLW-E $21 / 5$, bLW$\mathrm{E}_{31 / 7}$, and bLW-E $\mathrm{E}_{32 / 6}$ monolayer lattice strips, we could precisely define a SA dot position relative to its corresponding tile in most cases. For example, in the bottom panel of Figure $3 \mathrm{C}(\mathrm{d})$, SA dots always sit on the longitudinal frames along the tube axis. For each $\mathrm{X}$-shaped tile with its center as a lattice point, the SA dot at overhang 2 to its two adjacent arms connected with $1 *-1$ via the acute 
angle is through the clockwise rotation. Thus, we refer that the bLW-E $\mathrm{E}_{21 / 5}$ tubes have the left-handed curvature. Otherwise, if they have the right-handed curvature, the rotation direction from the SA-located overhang 2 to its two adjacent arms via the acute angle would be counterclockwise. Furthermore, we assigned that $c_{2}$-frames via the connection of $2 *-2$ aligned parallel to the tube axis, and $c_{1}$-frames via the connection of $1 *_{-} 1$ curled around the tube axis with left-handed rolling. In summary, we assayed over 60 individual bLW-E $E_{21 / 5}$ tubes at high-resolution from more than 5 batches with biotin/SA labels on overhang $1,1^{*}$, or 2 , all imaging results supported the left-handed curvature (more AFM images are in Figure S15).

Quantitative analysis of the curvature angle of a specific tube relies on assigning the chiral indices (n,m) to the tube. In practice, we could assign $(n, m)$ to a perforated bLW- $\mathrm{E}_{\mathrm{p} / \mathrm{q}}$ tube based on its high-resolution AFM images illustrating the tube lattices in both the open and the closed forms, such as in Figures S5-9. The high-resolution AFM images indicated that around 90\% of the bLW-E21/5 tubes had 4, 5, or 6 tube-axis-parallel $c_{2}$-frames from the bottom layer stuck on the mica, and a few other irregular tubes, around $10 \%$, had $c_{2}$-frames either clearly tilted against the tube axis or had wider perimeters. By directly counting (n,m) from a highly resolved tube in both open and closed forms (Figures S4-9), we were able to assign tubes with 4 bottom $c_{2}$-frames stuck on the mica mostly to $l-(6,2), l-(6,3)$, and $l-(7,3)$ (Figures S5, S10 and Table S2), 5 bottom $c_{2}$-frames mostly to $l-(8,3), l-(8,4)$, and $l-(9,4)$ (Figures S6, S7, S10, and Table S2), and 6 bottom $c_{2}$-frames to $l$-(10,4), $l$-(10,5), and $l-(11,5)$ (Figures S8, S9, S10, and Table S2). We also applied the numerical approximation method (Section S3-5, Table S2, and Figure S10) to match the chiral indices to the tube perimeter windows of Figure $2 \mathrm{C}(\mathrm{c})$. The numerical calculations illustrated that the bLW-E $\mathrm{E}_{21 / 5}$ tubes in Figure $2 \mathrm{C}(\mathrm{c})$ could be represented with a paraxial cluster of the chiral indices $(n, m)$ along the linear segment $n=2 m+1$, where $m$ is 3,4 and 5 in the $2 \mathrm{D}$ Cartesian coordinates $(\mathrm{m}, \mathrm{n})$ (Figure S10). The curvature angle range was estimated from these clustered tubes, which was sufficient to support our claim of semi-quantitation of the tile curvature. We took the most abundant bLW-E $21 / 5$ tube $l$-(8,4) (Figure $2 \mathrm{C}(\mathrm{c})$, Table S2 and Figure S10), as an example to describe the quantitation approach. First, we define the curvature of a tile as the bending of two halves of a tile along an axis passing the tile center to a dihedral, which could clearly be compared to a butt hinge with a pair of leaves bending along a shaft. It is reasonable to assume that the arms along $c_{1}$ in the bLW-E $E_{21 / 5} l-(8,4)$ tube bend at their centers of sticky end cohesion sites with the same curvature as the tile core. Thus, we approximated the transverse section of the perforated tube $l$ - $(8,4)$ by projecting its tube unit cell in Figure 2B to a 16-gon, as shown in Figure 2C(e). The physical pictures for the 16-gon were recognized as follows: 8 tile centers (lattice points) and 8 arm centers (lattice bond centers) along $c_{1}$ are projected to 16 vertices, and 16 straight half-arms (half lattice bonds) along $\boldsymbol{c}_{1}$ to 16 edges. Therefore, the curvature angle $\theta$ of $l-(8,4)$ was estimated as the exterior angle $360^{\circ} / 16=$ $22.5^{\circ}$, schematically shown in Figure 2B(f). Similarly, a 12-gon was approximated from the narrowest tube $l$ - $(6,3)$ and a 24 -gon from the widest tube $l-(12,6)$, and their curvature angles were estimated as $30^{\circ}$ and $15^{\circ}$, separately. From the above approximation approaches, the intrinsic tile curvature angle of bLW- $\mathrm{E}_{21 / 5}$ was estimated to be limited in the window of $22.5^{0} \pm 8^{0}$.

Furthermore, we investigated the evolution of the global tile curvature in both bLW-E $\mathrm{E}_{20 / 4}$ and bLW-E $\mathrm{E}_{22 / 6}$ tubes, which have 1 bp deletion and $1 \mathrm{bp}$ insertion from bLW- $\mathrm{E}_{21 / 5}$ in both arms and sticky ends, respectively. The biotin/SA labeling results (Figure S16) showed that bLW-E $E_{20 / 4}$ tubes possessed a left-handed curvature (6 individual tubes from 2 batches were measured), whereas bLW$\mathrm{E}_{22 / 6}$ tubes (Figure $\mathrm{S} 17$ ) had a right-handed curvature (6 individual tubes from 2 batches were measured). The unique bLW-E $\mathrm{E}_{20 / 4}$ tube was assigned to the chiral indices $l-(4,2)$, and the typical bLW-E $22 / 6$ tube to $r-(6,6)$. The opposite curvatures in bLW-E $20 / 4$ and bLW$\mathrm{E}_{22 / 6}$ tubes can be explained as follows: 1) The arm in bLW-E $\mathrm{E}_{20 / 4}$ with a helical twist density of $10.0 \mathrm{bp} /$ turn (less than $10.5 \mathrm{bp} / \mathrm{turn}$ of the natural B-DNA) and $4 \mathrm{nt}$ sticky end cohesion would generate a left-handed arm twist, whereas the arm in bLW-E2/6 with a helical twist density of $11.0 \mathrm{bp} /$ turn (larger than $10.5 \mathrm{bp} / \mathrm{turn}$ ) and $6 \mathrm{nt}$ sticky end cohesion would yield a right-handed arm twist. 2) Depending on coupling of the arm twist and the intrinsic tile curvature, bLW- $\mathrm{E}_{20 / 4}$ would enhance the left-handed curling to the unique tube $l$-(4,2) (Figure S16), while bLW-E $22 / 6$ would flip over the intrinsic tile curvature from left-handed to right-handed, thus resulting in tubes $r$ - $(5,5), r-(6,6)$, and $r-(7,7)$, among which $r-(6,6)$ was the most abundant one (Figure S17). Both tubes coexisted with many other residual fragments of tile oligomers, which indicated that the deviation of $\pm 1 \mathrm{bp}$ of arms from the natural B-DNA would disturb the tube formation process to some degree. In the bLW-E $\mathrm{E}_{2 / 4}$ tube of $l-(4,2), \boldsymbol{c}_{1}$ and $\boldsymbol{c}_{2}$-frames played their respective framing roles as 
in bLW-E $E_{21 / 5}$ (i.e., $\boldsymbol{c}_{1}$-frames curled around the tube axis with left-handed rolling and $\boldsymbol{c}_{2}$-frames aligned nearly parallel to the tube axis). For bLW-E $\mathrm{E}_{22 / 6}$ tubes of $r-(5,5)$ to $r$ - $(7,7)$, both $c_{1}$ - and $c_{2}$-frames were symmetric and played equal framing roles.

We also extended the bLW- $\mathrm{E}_{\mathrm{p} / \mathrm{q}}$ assemblies to the arm length of three full turns and the sticky end length from 5 to 7 nt. We tested three assemblies of bLW-E $\mathrm{E}_{31 / 5}, \mathrm{bLW}-\mathrm{E}_{31 / 7}$, and bLW-E $32 / 6$. For bLW-E $\mathrm{E}_{31 / 5}$ assemblies, the helical twist density of $31 / 3=10.3 \mathrm{bp} / \mathrm{turn}$ (less than $10.5 \mathrm{bp} /$ turn) in the arm and $5 \mathrm{nt}$ sticky end cohesion would generate a left-handed arm twist. Similar to bLW-E $\mathrm{E}_{20 / 4}$, the coupling of both the left-handed twisting forces from the arms and the tile cores generated the typical tube with the chiral indices $l$ $(10,5)$, where the left-handed curvature was defined from the biotin/SA labeling results ( 8 individual tubes from 2 batches were measured) and the yield for tube products was moderate (Figure S18).

The bLW-E $\mathrm{E}_{31 / 7}$ assemblies gave high yield products of uniform tubes with the chiral indices $(3,3)$, as shown in Figure 2D(b) and in block 1 of Figure S19. Investigation of the tile conformation with biotin/SA labeling was not as straightforward as for the bLW-E $21 / 5$ tubes because the bLW-E $\mathrm{E}_{31 / 7}$ tubes were much narrower. Controlling the blow of compressed air to generate proper monolayer structures for tile conformation assignment was challenging. We list a few difficulties that we encountered here: 1) When the tubes were broken by much stronger air-blowing, the open monolayer strips seldom bound SAs because labeled biotins were most probably blown to stick tightly on the mica, 2) softer blowing of compressed air couldn't properly open the tubes well because of the tightly bonded and narrow tube structure with the stronger sticky end cohesion of $7 \mathrm{nt}, 3$ ) even properly open monolayer strips were often discontinuous and their maximum strip width of 3 to 4 tiles (lattice points) in either $c_{1}$ or $c_{2}$ direction was the minimum threshold for tile conformation assignment. We tried to image more than a hundred of individual tubes, but were only able to clearly define 15 tubes' configuration with the right-handed curvature from 5 more batch assemblies, as shown in Figure 2D(c) and in block 2 and 3 of Figure S19. With the regular polygon approximation method, projection of the $r$ - $(3,3)$ tube unit cell elements to its transverse section could be approximated with a 12-gon, and the exterior angle (or the global tile curvature angle) was $30^{\circ}$. The global tile curvature of the bLW-E $\mathrm{E}_{31 / 7}$ tube of $r-(3,3)$ is schematically shown in Figure $2 \mathrm{D}(\mathrm{d})$, with the dihedral axis along $\boldsymbol{a}$.

Schematic and real bLW-E $\mathrm{E}_{32 / 6}$ tubes and their curvatures are shown in Figures 2E(a-e). Much different from previously assembled tubes with similar configurations, two types of tubes were achieved with high yield and high quality (a very few lattice fragments were imaged on mica), the "large" tube (bottom of Figure 2E(a) and its real images at the bottom of Figure 2E(b) and in block 1 of Figure S20) with its axis along $\boldsymbol{a}$ having an average perimeter of $407.1 \mathrm{~nm}$, represented with the chiral indices (16,16), and the "giant" tube (top of Figure 2E(a) and its real images at the top of Figure 2E(b) and in block 1 of Figure S20) with its axis along $\boldsymbol{b}$ having an average perimeter of $970.8 \mathrm{~nm}$, represented with the chiral indices $(23,-23)$. Because their tube widths and lengths were large enough to form well organized 2D monolayer lattices in both lateral and longitudinal directions, their tile conformations could be easily defined with the biotin/SA labeling technique. "Giant” tubes had the left-handed curvature (Figure 2E(c) and block 3 of Figure S20), whereas "large" tubes possessed the right-handed curvature (Figure 2E(d) and block 2 of Figure S20) (over 20 individual tubes of each type from 3 more batches were imaged). The global tile curvature angle of the "large" tube $r$ - $(16,16)$ was estimated as $9^{0}$ from a regular 64-gon (bottom of Figure 2E(e)), and that of the "giant" tube $l-(23,-23)$ as $3.9^{0}$ from a regular 92-gon (top of Figure 2E(e)). The widest "giant" bLW-E $\mathrm{E}_{32 / 6}$ tube reached up to $1600.0 \mathrm{~nm}$ in circumference and 5.0-8.0 $\mu \mathrm{m}$ in length, commensurate in width and even longer in length when compared to most of the well-behaved planar lattices assembled from small tiles via O-tiling.

For the bLW-E $\mathrm{E}_{32 / 6}$ assemblies, "large" tubes with the right-handed curvature further support the flip-over effect on the intrinsic lefthanded curvature of the bLW tile core. "Giant" tubes keep the left-handed curvature, while growing with their tube axes along $\boldsymbol{b}$, which are not thermodynamically favored. We propose the following mechanism to simultaneously grow both types of tubes: 1) Both arm length of $32 \mathrm{bp}$ with a helical twist density of $32 / 3=10.7 \mathrm{bp} /$ turn $(>10.5 \mathrm{bp} /$ turn of the natural B-DNA) and 6 nt sticky end cohesion would generate an integral right-handed twisting force. 2) The intrinsic tile curvature of bLW cores would generate a lefthanded curving force. 3) There should be a delicate balance between the two opposite curving forces at the initial assembly stage, which drives the tile oligomers to a saddle-like shape (Section S6 and Figure S11). In the saddle-like oligomers, the deeper curvature 
surrounding $\boldsymbol{a}$ is driven by the right-handed curving force and the other shallower curvature surrounding $\boldsymbol{b}$ is driven by the left-handed curving force. 4) With further growth, depending on which curved surface closes first to form a ring, the ring, as a tube seed, will end up growing epitaxially to a full tube. The saddle-like model of the tile oligomers is strongly supported by two evidences: 1) The tube growth directions or tube axes ("large" tubes along $\boldsymbol{a}$ and "giant" ones along $\boldsymbol{b}$ ) are perpendicular to each other. 2) The global tile curvatures ("large" tubes with the right-handed curvature and "giant" ones with the left-handed one) are opposite to each other. We name the specifically paired group of "large" and "giant" tubes as "orthogonal" tubes. The tube widening phenomena with $\boldsymbol{b}$ as the tube axis has also been observed in DAE-E tubes. However, according to the authors' descriptions, the tube formation mechanism and tube configuration were not very clear. ${ }^{35}$ In our case, the saddle-like oligomer model reasonably explains how "giant" tubes overcome the higher energy barrier to form a ring seed and finally grow up epitaxially with their tube axes along $\boldsymbol{b}$.

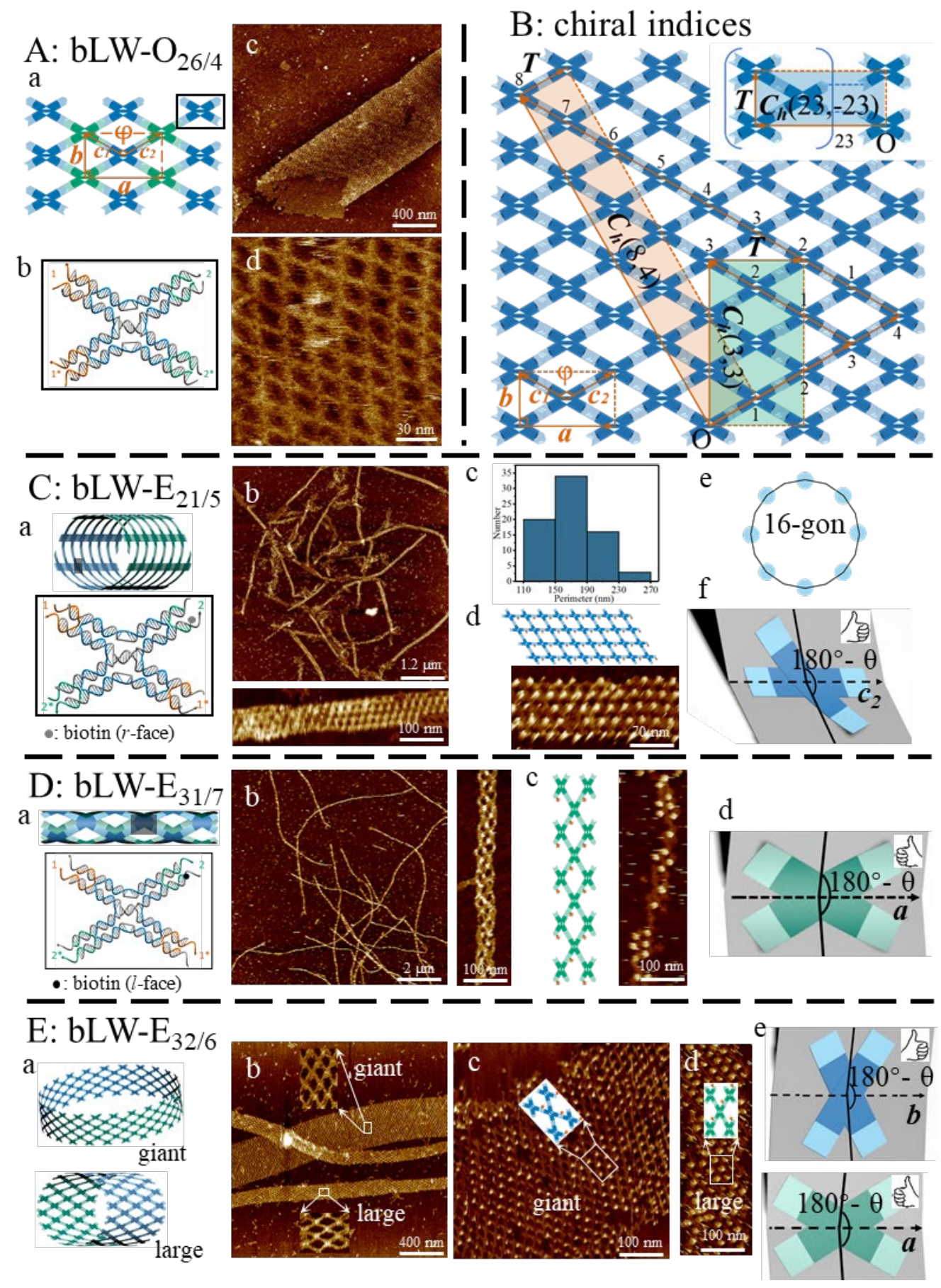


Figure 2. Bridging longitudinal weaving and tiling systems and tile curvature transformation. (A) bLW-O $26 / 4$ : a) an assembly model with tile brick modules, b) a helix tile model, c and d) zoomed-out and zoomed-in AFM images. (B) Three sets of chiral indices representing typical E-tiling tubes: a typical bLW-E21/5 tube unit cell of $(8,4)$ shadowed in gold with its tube axis along $c_{2}$, the unique bLW-E31/7 tube unit cell of $(3,3)$ shadowed in green with its tube axis along $a$, and a "giant" bLW-E $32 / 6$ tube unit cell of $(23,-23)$ shadowed in blue with its tube axis along $b$. (C) bLW-E $21 / 5$ : a) a brick tube model of $l$ - $(8,4)$ with $l$-faces colored blue inside and $r$-faces colored green outside (top) and a helix tile model (bottom) with a biotin label at overhang 2 pointing to the tile's $r$-face, b) zoomed-out (top) and zoomed-in (bottom) AFM images of bLW-E $21 / 5$ tubes, c) perimeter distribution of 73 tubes, d) a brick assembly model carrying biotin/SA labels (top) and its corresponding zoomed-in AFM image (bottom) of an inside surface lattice with biotin/SA dots, e) approximation of the transverse section of tube $l$ - $(8,4)$ with 16-gon, f) the intrinsic tile curvature represented with a dihedral along the axis $c_{2}$ following the left-hand grip rule. (D) bLWE31/7 tubes: a) a brick tube model of $r$ - $(3,3)$ with $l$-faces in blue outside and $r$-faces in green inside (top) and a helix tile model with a biotin label at overhang 2 (bottom) pointing to the tile's $l$-face, b) zoomed-out (left) and zoomed-in (right) AFM images, c) a brick assembly model carrying biotin/SA labels (left) and its corresponding zoomed-in AFM image (right) of an inside surface lattice with biotin/SA dots, d) the global tile curvature of $r$ - $(3,3)$ represented with a dihedral along the axis $a$ following the right-hand grip rule. E, bLW-E32/6 tubes: a) a brick model of a "giant" tube $l-(23,-23)$ (top) and that of a "large" tube $r-(16,16)$ (bottom), and the helix tile model of bLW-E32/6 with a biotin/SA label at overhang 2 is similar to bLW-E $31 / 7$ and thus omitted, b) AFM images of both "large" and "giant" tubes and their corresponding zoomed-in images in insets illustrating their different tube axes, c) a zoomed-in AFM image of the inside surface lattice of a "giant" tube with biotin/SA dots and its corresponding $l$-faced brick model (inset), d) a zoomed-in AFM image of an inside surface lattice of a "large" tube with biotin/SA dots and its corresponding $r$-faced brick model (inset), e) the global tile curvature of the "giant" tube (top) represented with a dihedral along the axis $b$ following the left-hand grip rule and that of the "large" tube (bottom) with a dihedral along the axis $a$ following the right-hand grip rule.

In Table 1, we summarize the 2D lattice linear and angular constants $(a, b, c$, and $\varphi)$ of all investigated bLW- $\mathrm{E}_{\mathrm{p} / \mathrm{q}}$ tubes, followed with the tube parameters of the chiral indices $(n, m)$, perimeter $(C)$, curvature angle $(\theta)$, and length range of the most abundant tube for each design.

Table 1. Experimental unit cell parameters and tube parameters of $b L W-E_{p / q}$ tubes. ${ }^{\#}$

\begin{tabular}{|c|c|c|c|c|c|c|c|c|}
\hline Tube & $a / \mathrm{nm}$ & $b / \mathrm{nm}$ & $c / \mathrm{nm}$ & $\varphi /{ }^{\circ}$ & $\boldsymbol{C h}(\mathrm{n}, \mathrm{m})$ & $\mathrm{C} / \mathrm{nm}$ & $\theta$ (axis) & length/ $\mu \mathrm{m}$ \\
\hline bLW-E $20 / 4$ & 34.4 & 21.9 & 20.4 & $115^{\circ}$ & $l-(4,2)$ & 74.3 & $45^{\circ}\left(c_{2}\right)$ & $2.0-5.0$ \\
\hline bLW-E $E_{21 / 5}$ & 35.1 & 23.7 & 21.2 & $112^{\circ}$ & $l-(8,4)$ & 158.8 & $22.5^{\circ}\left(c_{2}\right)$ & $3.0-6.0$ \\
\hline $\mathrm{bLW}-\mathrm{E}_{22 / 6}$ & 36.3 & 22.7 & 21.4 & $116^{\circ}$ & $r-(6,6)$ & 136.2 & $15^{\circ}(a)$ & $2.0-5.0$ \\
\hline bLW-E $E_{31 / 5}$ & 41.2 & 25.7 & 24.3 & $116^{\circ}$ & $l-(10,5)$ & 219.0 & $18^{\circ}\left(c_{2}\right)$ & $4.0-7.0$ \\
\hline $\mathrm{bLW}-\mathrm{E}_{31 / 7}$ & 41.9 & 24.2 & 24.2 & $120^{\circ}$ & $r-(3,3)$ & 72.7 & $30^{\circ}(a)$ & $6.0-10.0$ \\
\hline $\begin{array}{l}\mathrm{bLW}-\mathrm{E}_{32 / 6} \\
\text { (giant) }\end{array}$ & 42.2 & 24.9 & 24.5 & $119^{\circ}$ & $l-(23,-23)$ & 970.8 & $4^{\circ}(\boldsymbol{b})$ & $4.0-8.0$ \\
\hline $\begin{array}{l}\text { bLW-E } E_{32 / 6} \\
\text { (large) }\end{array}$ & 42.3 & 25.4 & 24.7 & $118^{\circ}$ & $r-(16,16)$ & 407.1 & $5.6^{\circ}(a)$ & $4.0-8.0$ \\
\hline
\end{tabular}

\footnotetext{
\# For all six designs, bLW-E $\mathrm{E}_{20 / 4}$ and bLW-E $\mathrm{E}_{31 / 7}$ have their corresponding unique tubes with the chiral indices $\boldsymbol{C h}(\mathrm{n}, \mathrm{m})$ of $l-(4,2)$ and $r-(3,3)$, respectively; bLW- $\mathrm{E}_{21 / 5}, \mathrm{bLW}-\mathrm{E}_{22 / 6}$, and bLW-E $\mathrm{E}_{31 / 5}$ are represented with their most abundant tubes $l-(8,4), r-(6,6)$, and $l-$ $(10,5)$, respectively; and the "giant" and "large" tubes in bLW-E $E_{32 / 6}$ are represented with $l-(23,-23)$ and $r$ - $(16,16)$ at their average perimeters, respectively. The tube perimeter $\mathrm{C}$ was calculated as $\mathrm{C}=c \sqrt{n^{2}+m^{2}+2 n m \cos \varphi}$, where $c$ and $\varphi$ measured from experiments are listed in the same row (refer to Section S3 of SI). The curvature angle $\theta$ is correlated with the tile dihedral axis, and
} 
was estimated according to the following approximation rule: For each $b L W-E_{p / q}$ tube represented with $(n, m)$, when $n=2 m, \theta$ was calculated as the exterior angle of a regular 2n-gon; when $n=|m|, \theta$ was calculated as the exterior angle of a regular $4 n-g o n$.

\section{D arrays of transverse weaving (TW) tiles}

Figures $3 \mathrm{~A}$ and $3 \mathrm{~B}$ show the transverse weaving of the $\mathrm{H}$-shaped c128nt scaffold with six helper strands to a stable tile core, and further with O-tiling to TW-O ${ }_{26 / 4}$ planar lattices and E-tiling to TW-E $21 / 5$ tubes. In the TW core, each helper strand weaves in the transverse direction over and under every other scaffold strand four times, forming the densest HJs in 2D DNA lattices with a density of $2 \mathrm{HJ} /$ turn. ${ }^{45}$ Compared with LW and bLW cores having 5 and $4 \mathrm{HJs}$, respectively, the TW core possesses 12 HJs.

The face-alternating O-tiling assembly of $\mathrm{TW}-\mathrm{O}_{26 / 4}$ provides a planar handkerchief-like lattice with dimensions up to $4 \times 4 \mu \mathrm{m}^{2}$, shown in Figures $3 \mathrm{~A}(\mathrm{c})$ and $\mathrm{S} 21$. To achieve high-resolution images with individual tile textures, as shown in Figure $3 \mathrm{~A}(\mathrm{~d})$ and in Figure S21, more than 4 scans were needed. However, repeated scans moved some tiles away and left dim pores illustrating tile shadows in the lattice clearly. In contrast with the $\mathrm{LW}-\mathrm{E}_{21 / 5}$ and $\mathrm{LW}-\mathrm{O}_{26 / 4}$ ribbon-like lattices having ragged edges (stepped faces) along their longitudinal directions, TW-O ${ }_{26 / 4}$ lattices presented sharp vertices and straight edges (flat faces) along $c_{1}$ and $c_{2}$, due to the strong bonds with three helixes integrated together as an arm for sticky end cohesion. ${ }^{46}$ As shown in Figure $3(\mathrm{~d})$, with assignments of $c_{1}$ and $\boldsymbol{c}_{2}$ along the sharp edges, $\boldsymbol{a}$ and $\boldsymbol{b}$ were defined easily.

E-tiling assembly of TW- $E_{21 / 5}$ shows relatively homogenous tubes (Figure 3B(b) and block 1 of Figure S22). Although the TW core has the highest HJ density, formation of tubes but not planar arrays evidenced that the TW core has an intrinsic curvature, probably due to the lack of a central HJ and thus the bending of both half-parts along $\boldsymbol{a}$ passing the TW core center. Similarly, we achieved their high-resolution AFM images with individual tile textures by repeated scans in the same region more than 4 times. As shown in Figure $3 \mathrm{~B}(\mathrm{c})$ and block 1 of Figure S22, parts of the top layers were torn open along the flat face of $\boldsymbol{c}_{1}$ or $\boldsymbol{c}_{2}$. The TW-E $21 / 5$ tube axis is along the arm helix extension direction of $\boldsymbol{a}$ because it is thermodynamically favored. The tile numbers along $\boldsymbol{c}_{1}$ and $\boldsymbol{c}_{2}$ in Figure $3 \mathrm{~B}$ (c) were counted to 10 , thus, this tube was assigned to the chiral indices $(5,5)$.

The tile conformation was defined with the in-situ biotin/SA labeling technique too. In contrast with the perforated bLW- $\mathrm{E}_{\mathrm{p} / \mathrm{q}}$ tubes, $\mathrm{TW}-\mathrm{E}_{21 / 5}$ assemblies were sealed tubes without leaving any space between the tiles. In this case, the biotin orientation on micasupported lattices was key for the in-situ binding of SAs. When biotins were exposed to the outside on the top of a DNA lattice, SAs bound to biotins efficiently, whereas when biotins were buried by a monolayer lattice, no binding occurred. We applied three biotinlabeling strategies: 1) only an iBiodT label at overhang 2 pointing to the $l$-face of every TW core, 2) only a biotin label at the 5 '-end of a helper strand at overhang $1^{*}$ pointing to the $r$-face of every TW core, 3 ) the two types of biotin labels at both $r$-and $l$-faces of every TW core, as shown in the TW- $\mathrm{E}_{21 / 5}$ tile helix model of Figure 3B(a) (bottom). After in-situ binding of SAs, the biotin labels exposing on $l$-faces bind SAs only on the top of double-layers (or sealed tubes), but not on monolayers (or open tubes), as shown in Figure 3B(d) and block 2 of Figure S22; the biotin labels exposing on $r$-faces bind SAs only on monolayers but not on the top of double-layers, as shown in Figure 3B(e) and block 3 of Figure S22; biotin labels exposing on both $r$ - and $l$-faces bind SAs on both monolayers and the top of double-layers, as shown in block 4 of Figure S22. For each labeling design, we have imaged more than 30 individual tubes in 3 batches. All imaging results support that the intrinsic tile curvature of TW-E $\mathrm{E}_{21 / 5}$ followed the right-hand grip rule.

We measured the tube perimeters of 67 tubes in more than 5 different batches by doubling the squashed tube width. The tube perimeter abundances are plotted in Figure 3B(g), indicating a nearly normal distribution from 45 to $135 \mathrm{~nm}$. Combining both the direct counting of the chiral indices $(\mathrm{n}, \mathrm{m})$ from high-resolution AFM images and the numerical approximation method in Section S3, we assigned the most abundant tube $(21 / 67=31.3 \%)$ at the perimeter window of $75.0 \sim 90.0 \mathrm{~nm}$ to $r-(6,6)$, the secondly most abundant tubes $(14 / 67$ $=21 \%)$ at $60.0 \sim 75.0 \mathrm{~nm}$ to $r-(5,5)$ and at $90.0 \sim 105.0 \mathrm{~nm}$ to $r-(7,7)$, and other less abundant tubes at $45.0 \sim 60.0 \mathrm{~nm}$ to $r-(4,4)(6 / 67=$ 9.0\%), at 105.0 120.0 nm to $r-(8,8)(9 / 67=13.4 \%)$, and at $120.0 \sim 135.0 \mathrm{~nm}$ to $r-(9,9)(3 / 67=4.5 \%)($ Section S5 and Table S3). Because TW-E $E_{21 / 5}$ tubes have all helixes juxtaposed closely without any space between them, and the arm helixes are parallel to the 
tube axis, there will be no bending occurring in the arm bonds. Therefore, we approximated the transverse section of the most abundant $r$ - $(6,6)$ to a regular 12-gon, that of the narrowest $r$ - $(4,4)$ to a regular 8 -gon, and that of the widest tube $r$ - $(9,9)$ to a 18 -gon. The curvature angle window was estimated to be around $30^{\circ} \pm 15^{\circ}$, which is schematically represented in Figure $3 \mathrm{~B}(\mathrm{f})$.

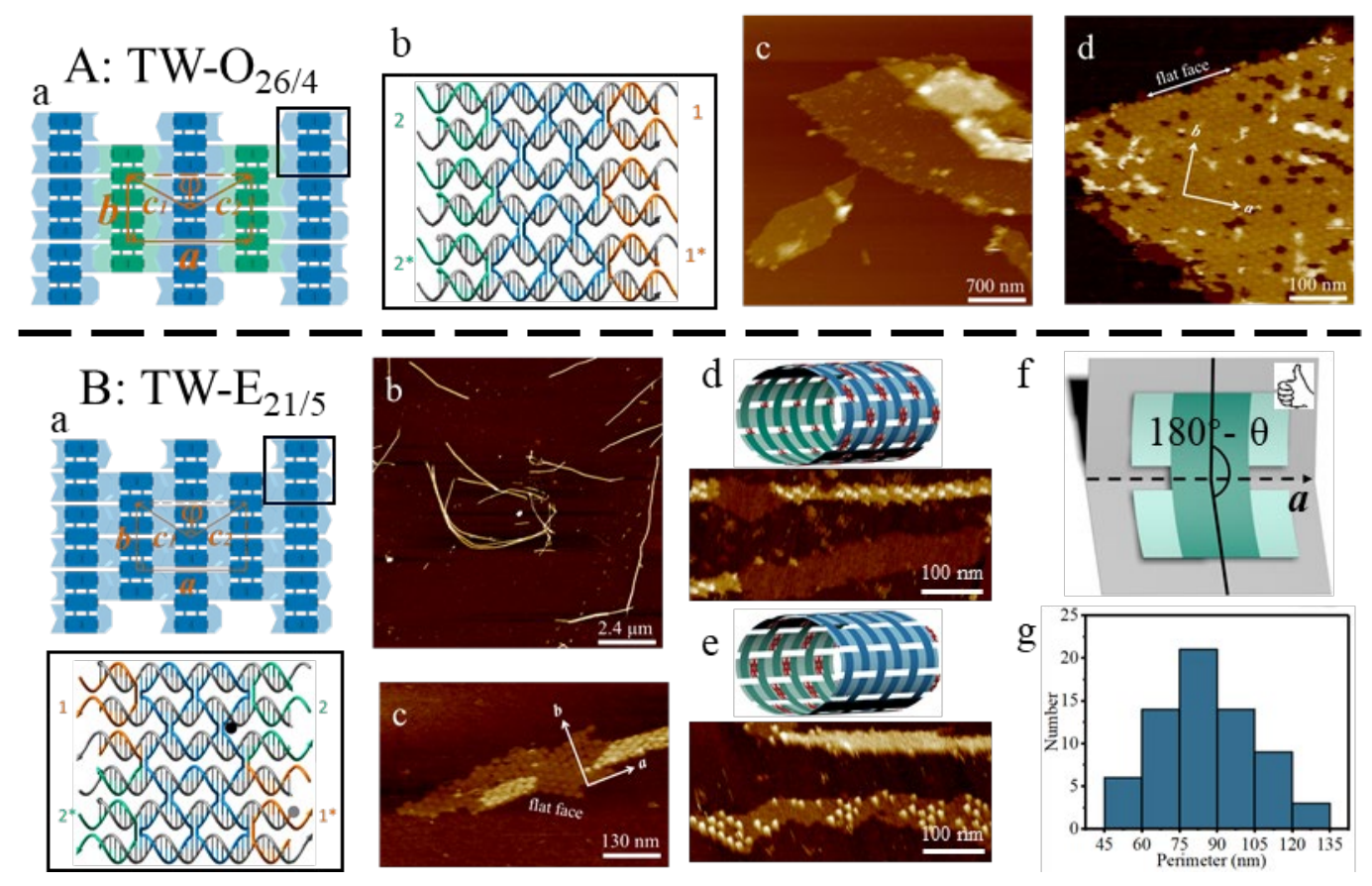

Figure 3. Transverse weaving and tiling systems. (A) TW- $\mathrm{O}_{26-4}$ : a) an assembly model with tile brick modules, b) a helix tile model, in which the two centered helixes are $20 \mathrm{bp}$ and the rest of four helixes (upper two and lower two helixes) are $22 \mathrm{bp}$ in length within the tile core along $a$, c and d) zoomed-out and zoomed-in AFM images with lattice constants of $a=30.6 \mathrm{~nm}, b=14.7 \mathrm{~nm}, c=17.0 \mathrm{~nm}$, and $\varphi=$

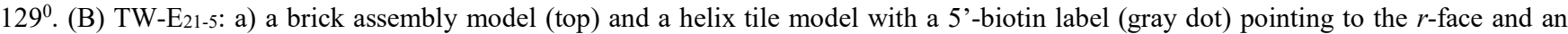
iBiodT (dark dot) pointing to the $l$-face (bottom), b) a zoomed-out image, c) a zoomed-in image with lattice constants of $a=27.3 \mathrm{~nm}, b=$ $14.3 \mathrm{~nm}, c=15.3 \mathrm{~nm}$, and $\varphi=128^{\circ}$, d) a brick assembly model of the tube $r$ - $(6,6)$ with biotin/SA labels on $l$-faces and its corresponding zoomed-in images with SA dots only bound on the top of double-layers, e) a brick assembly model of the tube $r$ - $(6,6)$ with biotin/SA labels on $r$-faces and its corresponding zoomed-in images with SA dots only bound on monolayers, $\mathrm{f}$ ) the intrinsic tile curvature of TW-E $21 / 5$ tubes represented with a dihedral along the axis $a$ following the right-hand grip rule, $(\mathrm{g}) 67$ tubes distributed in 6 perimeter windows.

\section{Comprehensive Discussion}

In this work, the most interesting phenomenon was the evolution of the global tile curvatures of bLW- $\mathrm{E}_{\mathrm{p} / \mathrm{q}}$ tubes with changing arm lengths at 4 or 6 half-turns and sticky end lengths in the range of 4 to $7 \mathrm{nt}$. As we showed, many parameters influence the global tile curvature such as the intrinsic tile curvature, the arm length, the sticky end length, the tile orientation versus the tube axis. We simplified the complicated factors into a model in which the tube configuration would be tuned with two twisting forces. One is resulted from the intrinsic tile curvature and the other from the arm twist, which was affected by both the arm and the sticky end lengths. The intrinsic tile curvature of a tile core depended on its weaving architecture. For example, bLW cores had the intrinsic lefthanded curvature, while TW cores possessed the intrinsic right-handed curvature, both of which were referred from the bLW-E $\mathrm{E}_{21 / 5}$ and TW-E $E_{21 / 5}$ tubes without any extra twist in the arm. The extra arm twist nearly follows the overwinding/underwinding rule ${ }^{22}$ by regulating the inter-tile arm lengths between two adjacent HJs, except for the assembly of bLW-E $\mathrm{E}_{31 / 7}$ tubes. To briefly describe the overwinding/underwinding rule, when the helical twist density in an arm is at $10.5 \mathrm{bp} /$ turn (precisely $10.44 \mathrm{bp} /$ turn) ${ }^{24}$ of the natural B-DNA, the arm would be straight; when it is less than $10.5 \mathrm{bp} /$ turn, the arm would gain a left-handed twisting torque; and when it 
is larger than $10.5 \mathrm{bp} /$ turn, the arm would gain a right-handed twisting torque. ${ }^{22}$ In our case, according to experimental results, the rule needed small modifications: When a sticky end cohesion exists in the arm and the sticky end length is at 4 or $5 \mathrm{nt}$, the extra twisting torque generated from the sticky end cohesion could be ignored, thus, the above overwinding/underwinding rule was respected; however when the sticky end length was at 6 and $7 \mathrm{nt}$, the extra right-handed twisting torque generated from the sticky end cohesion needed to be counted. ${ }^{47-48}$ Taking bLW-E $E_{31 / 7}$ tubes as the example, although its helical twist density of the arm of $31 / 3=$ $10.3 \mathrm{bp} /$ turn is less than $10.5 \mathrm{bp} / \mathrm{turn}$, its much stronger $7 \mathrm{nt}$ sticky end cohesion could generate an extra right-handed twisting torque, thus, coupling of the right-handed arm twist and the intrinsic left-handed tile curvature generated the unique bLW-E $\mathrm{E}_{31 / 7}$ tube $r$ - $(3,3)$ in high yield with the right-handed curvature.

We grew tubes with a very slow annealing process over $70 \mathrm{~h}$, thus, all lattice structures were thermodynamically controlled. From our AFM imaging results, we found out that bLW- $\mathrm{E}_{\mathrm{p} / \mathrm{q}}$ tubes always presented either one of two clusters of the chiral indices (n,m) with 1 ) $\mathrm{n} \approx 2 \mathrm{~m}$ and 2 ) $\mathrm{n} \approx \mathrm{m}$ when $0 \leq \mathrm{m} \leq \mathrm{n}$. When $\mathrm{n} \approx 2 \mathrm{~m}$, tubes possessing the left-handed curvature were governed by the intrinsic tile curvature, while when $\mathrm{n} \approx \mathrm{m}$, tubes following the right-handed curvature were controlled by the extra right-handed arm twist. The flip-over of tile curvature could be compared to that of a contact lens. We suggest that the bLW tile core is always curved due to its rigidity, and its intrinsic left-handed curvature can be forced by stronger opposite arm twists to switch to the right-handed curvature during assembly. The two types of chiral indices $(\mathrm{n}, \mathrm{m})$ of $\mathrm{n} \approx 2 \mathrm{~m}$ and $\mathrm{n} \approx \mathrm{m}$ should represent the most stable structures with global energy minima for tubes possessing the left-handed and right-handed curvatures, respectively. Other intermediate tubes with $\mathrm{n}$ and $\mathrm{m}(0 \leq \mathrm{m} \leq \mathrm{n})$ deviating from $\mathrm{n} \approx 2 \mathrm{~m}$ and $\mathrm{n} \approx \mathrm{m}$ should possess higher deformation energies and would not be thermodynamically favored. In fact, we imaged a few of these tubes in the bLW-E ${ }_{21 / 5}$ assemblies, but they were less than $5 \%$ of the total. In all the tested bLW-E $\mathrm{E}_{\mathrm{p} / \mathrm{q}}$ tubes, we only achieved three tube assemblies in high yield, bLW-E $21 / 5, \mathrm{bLW}-\mathrm{E}_{31 / 7}$, and bLW-E $\mathrm{E}_{32 / 6}$. Thus, the tube assembly performance depends on a delicate balance between the intrinsic tile curvature and arm twists, and also on the helix winding phase and geometry match between tile cores and arms.

Formation of nonhomogeneous tubes assembled from small tiles by O-tiling is often interpreted as the decrease of the global free energy of the system. We observed dominant tubular structures and minor planar lattices in bLW-O ${ }_{26 / 4}$ assemblies, which might be attributed to the rigidity of bLW tile cores always possessing a curvature. The curvature flip-over effect observed in E-tiling tubes might also occur in O-tiling assemblies. Once a ring seed spontaneously forms, epitaxial extension of the tube lattices could force the oppositely curled tiles in bLW-O $\mathrm{O}_{26 / 4}$ assemblies to switch to the same curled ones. As for the less rigid TW and DX tiles, the intrinsic tile curvature is more flexible, thus, O-tiling of TW and DAE tiles generates dominant planar ribbons and minor nonhomogeneous tubes with random stoichiometric ratios, suggesting that the global curling bias is much weaker on the TW-O assemblies $^{2}$ than on the bLW-O $26 / 4$ ones. Overall, coupling of shallower intrinsic tile curvatures with weaker arm twisting torques would generate larger lattices or wider tubes easily in higher probability, whereas coupling of deeper intrinsic curvatures with stronger arm twisting torques to generate arrays with large dimensions would be difficult to achieve, and most probably would yield illbehaved fragments of tile oligomers, narrower tubes, or even fibers rather than well-behaved DNA lattices. Other couplings between the two extremes would generate moderate structures in a complicated way.

In conclusion, we not only realized three weaving architectures to construct three new tile cores, LW, bLW, TW, and their 2D lattices, but also deciphered each tube configuration and thus their component tiles conformation with the biotin/SA labeling technique. On the basis of abstracting the 2D Bravais lattice of centered rectangle for each assembly, we introduced the chiral indices to quantify the global tile curvature. With a simplified model that we used to analyze in details the curving forces acting on DNA tubes into two types, the intrinsic tile curving force and the extra arm curving force, formation of the DNA tubes to a plethora of different shapes, diameters, and configurations can be explained quite well by the coupling of both forces. Such detailed investigation of DNA tube curvatures including structure and quantitation will be helpful in the future for engineering DNA nanostructures with high yield and high quality on one hand, and also for investigating the different physicochemical properties and biological functionalities of DNA chiral assemblies on the other hand. 


\section{ASSOCIATED CONTENT}

Supporting Information available. Supporting Information Available: experimental methods, a discussion about the theoretical estimation of lattice linear and angular constants, a discussion about the tube parameters calculated from the chiral indices ( $\mathrm{n}, \mathrm{m})$, a discussion about the assignment of the chiral indices $(n, m)$ to a specific tube based on its high-resolution AFM image, a discussion of assignments of a cluster of chiral indices by the numerical approximation method to both bLW-E $21 / 5$ and TW-E21/5 tubes, a discussion of the saddle-like tile oligomer model to simultaneously grow "large" and "giant" tubes, additional AFM images of each assembly with descriptions in its figure caption, and sequence information. These materials are available free of charge via the Internet.

\section{AUTHOR INFORMATION}

\section{Corresponding Author}

Nadrian C. Seeman - Department of Chemistry, New York University, New York, NY 10003, USA

https://orcid.org/0000-0002-9680-4649;

Email : ned.seeman@nyu.edu

Shou-Jun Xiao - State Key Laboratory of Coordination Chemistry, School of Chemistry and Chemical Engineering, Nanjing

University, Nanjing 210093, Jiangsu, China.

https://orcid.org/0000-0002-4240-1852;

Email:sjxiao@nju.edu.cn

\section{Author Contributions}

${ }^{\circ} \mathrm{CJ}$ and BL contributed equally. / All authors have given approval to the final version of the manuscript. /

\section{Notes}

The authors declare no competing financial interest.

\section{ACKNOWLEDGMENT}

SJX acknowledges the financial support from the National Natural Science Foundation of China (Grant No. 91753134, 21571100). NCS acknowledges the financial support from Grant No. GM-29554 from NIGMS, Grant Nos. CTS1120890, CCF-1117210, EFRI-1332411, and CHE-1708776 from the NSF, Grant No. W911NF-1110024 from ARO, Grant Nos. N000141110729 and N000140911118 from ONR, DESC0007991 from DOE for partial salary support and GBMF3849 from the Gordon and Betty Moore Foundation.

\section{REFERENCES}

(1) Seeman, N. C., DNA in a Material World. Nature 2003, 421 (6921), 427-431.

(2) Winfree, E.; Liu, F.; Wenzler, L. A.; Seeman, N. C., Design and Self-Assembly of Two-Dimensional DNA Crystals. Nature 1998, 394 (6693), 539-544.

(3) Rothemund, P. W. K., Folding DNA to Create Nanoscale Shapes and Patterns. Nature 2006, 440 (7082), $297-302$.

(4) Seeman, N. C.; Sleiman, H. F., DNA Nanotechnology. Nat. Rev. Mater. 2017, 3 (1), 17068.

(5) Hong, F.; Zhang, F.; Liu, Y.; Yan, H., DNA Origami: Scaffolds for Creating Higher Order Structures. Chem. Rev. 2017, 117 (20), 12584-12640.

(6) Ramezani, H.; Dietz, H., Building Machines with DNA Molecules. Nat. Rev. Genet. 2020, 21 (1), 5-26.

(7) Aldaye, F. A.; Palmer, A. L.; Sleiman, H. F., Assembling Materials with DNA as the Guide. Science 2008, 321 (5897), $1795-1799$.

(8) Platnich, C. M.; Hariri, A. A.; Sleiman, H. F.; Cosa, G., Advancing Wireframe DNA Nanostructures Using Single-Molecule Fluorescence Microscopy Techniques. Acc. Chem. Res. 2019, 52 (11), 3199-3210.

(9) Fu, T.; Seeman, N., DNA Double-Crossover Molecules. Biochemistry 1993, 32 13, 3211-20. 
(10) Liu, F.; Sha, R.; Seeman, N. C., Modifying the Surface Features of Two-Dimensional DNA Crystals. J. Am. Chem. Soc. 1999, 121 (5), 917-922.

(11) Mao, C.; Sun, W.; Seeman, N. C., Designed Two-Dimensional DNA Holliday Junction Arrays Visualized by Atomic Force Microscopy. J. Am. Chem. Soc. 1999, 121 (23), 5437-5443.

(12) Zheng, J.; Birktoft, J. J.; Chen, Y.; Wang, T.; Sha, R.; Constantinou, P. E.; Ginell, S. L.; Mao, C.; Seeman, N. C., From Molecular to Macroscopic via the Rational Design of a Self-Assembled 3D DNA Crystal. Nature 2009, 461 (7260), 74-77.

(13) Liu, D.; Wang, M.; Deng, Z.; Walulu, R.; Mao, C., Tensegrity: Construction of Rigid DNA Triangles with Flexible Four-Arm DNA Junctions. J. Am. Chem. Soc. 2004, 126 (8), 2324-2325.

(14) Shih, W. M.; Quispe, J. D.; Joyce, G. F., A 1.7-Kilobase Single-Stranded DNA that Folds into a Nanoscale Octahedron. Nature 2004, 427 (6975), 618-621.

(15) Zhang, C.; He, Y.; Chen, Y.; Ribbe, A. E.; Mao, C., Aligning One-Dimensional DNA Duplexes into Two-Dimensional Crystals. J. Am. Chem. Soc. 2007, 129 (46), 14134-14135.

(16) Yin, P.; Hariadi, R. F.; Sahu, S.; Choi, H. M. T.; Park, S. H.; LaBean, T. H.; Reif, J. H., Programming DNA Tube Circumferences. Science 2008, 321 (5890), 824-826.

(17) Ke, Y.; Ong, L. L.; Shih, W. M.; Yin, P., Three-Dimensional Structures Self-Assembled from DNA Bricks. Science 2012, 338 (6111), $1177-1183$

(18) Yan, H.; Park, S. H.; Finkelstein, G.; Reif, J. H.; LaBean, T. H., DNA-Templated Self-Assembly of Protein Arrays and Highly Conductive Nanowires. Science 2003, 301 (5641), 1882-1884.

(19) Tian, C.; Li, X.; Liu, Z.; Jiang, W.; Wang, G.; Mao, C., Directed Self-Assembly of DNA Tiles into Complex Nanocages. Angew. Chem. Int. Ed. 2014, 53 (31), 8041-8044.

(20) Guo, X.; Wang, X.-M.; Wei, S.; Xiao, S.-J., Construction of a Holliday Junction in Small Circular DNA Molecules for Stable Motifs and Two-Dimensional Lattices. ChemBioChem 2018, 19 (13), 1379-1385.

(21) Guo, X.; Wang, X. M.; Xiao, S. J., Stable DNA Motifs, 1D and 2D Nanostructures Constructed from Small Circular DNA Molecules. J. Vis. Exp. 2019, (146).

(22) Dietz, H.; Douglas, S. M.; Shih, W. M., Folding DNA into Twisted and Curved Nanoscale Shapes. Science 2009, 325 (5941), 725730 .

(23) Ke, Y.; Douglas, S. M.; Liu, M.; Sharma, J.; Cheng, A.; Leung, A.; Liu, Y.; Shih, W. M.; Yan, H., Multilayer DNA Origami Packed on a Square Lattice. J. Am. Chem. Soc. 2009, 131 (43), 15903-15908.

(24) Woo, S.; Rothemund, P. W., Programmable Molecular Recognition Based on the Geometry of DNA Nanostructures. Nat. Chem. 2011, $3(8), 620-7$.

(25) Maier, A. M.; Bae, W.; Schiffels, D.; Emmerig, J. F.; Schiff, M.; Liedl, T., Self-Assembled DNA Tubes Forming Helices of Controlled Diameter and Chirality. ACS Nano 2017, 11 (2), 1301-1306.

(26) Wei, B.; Dai, M.; Myhrvold, C.; Ke, Y.; Jungmann, R.; Yin, P., Design Space for Complex DNA Structures. J. Am. Chem. Soc. 2013, 135 (48), 18080-18088.

(27) Marchi, A. N.; Saaem, I.; Vogen, B. N.; Brown, S.; LaBean, T. H., Toward Larger DNA Origami. Nano Lett. 2014, 14 (10), 5740 5747.

(28) Benn, F.; Haley, N. E. C.; Lucas, A. E.; Silvester, E.; Helmi, S.; Schreiber, R.; Bath, J.; Turberfield, A. J., Chiral DNA Origami Nanotubes with Well-Defined and Addressable Inside and Outside Surfaces. Angew. Chem. Int. Ed. 2018, 57 (26), 7687-7690.

(29) Sun, S.; Yang, Y.; Li, D.; Zhu, J., Large Chiral Nanotubes Self-Assembled by DNA Bricks. J. Am. Chem. Soc. 2019, 141 (50), 1952419528.

(30) Mathieu, F.; Liao, S.; Kopatsch, J.; Wang, T.; Mao, C.; Seeman, N. C., Six-Helix Bundles Designed from DNA. Nano Lett. 2005, 5 (4), 661-665.

(31) Hong, F.; Jiang, S.; Lan, X.; Narayanan, R. P.; Šulc, P.; Zhang, F.; Liu, Y.; Yan, H., Layered-Crossover Tiles with Precisely Tunable Angles for 2D and 3D DNA Crystal Engineering. J. Am. Chem. Soc. 2018, 140 (44), 14670-14676. 
(32) Qian, H.; Tian, C.; Yu, J.; Guo, F.; Zheng, M.-S.; Jiang, W.; Dong, Q.-F.; Mao, C., Self-Assembly of DNA Nanotubes with Defined Diameters and Lengths. Small 2014, 10 (5), 855-858.

(33) Mitchell, J. C.; Harris, J. R.; Malo, J.; Bath, J.; Turberfield, A. J., Self-Assembly of Chiral DNA Nanotubes. J. Am. Chem. Soc. 2004, $126(50), 16342-16343$.

(34) Liu, X.; Zhao, Y.; Liu, P.; Wang, L.; Lin, J.; Fan, C., Biomimetic DNA Nanotubes: Nanoscale Channel Design and Applications. Angew. Chem. Int. Ed. Engl. 2019, 58 (27), 8996-9011.

(35) Rothemund, P. W. K.; Ekani-Nkodo, A.; Papadakis, N.; Kumar, A.; Fygenson, D. K.; Winfree, E., Design and Characterization of Programmable DNA Nanotubes. J. Am. Chem. Soc. 2004, 126 (50), 16344-16352.

(36) Ke, Y.; Liu, Y.; Zhang, J.; Yan, H., A Study of DNA Tube Formation Mechanisms Using 4-, 8-, and 12-Helix DNA Nanostructures. J. Am. Chem. Soc. 2006, 128 (13), 4414-4421.

(37) Sherman, W. B.; Seeman, N. C., Design of Minimally Strained Nucleic Acid Nanotubes. Biophys. J. 2006,90 (12), $4546-4557$.

(38) Wang, T.; Schiffels, D.; Cuesta, S. M.; Fygenson, D. K.; Seeman, N. C., Design and Characterization of 1D Nanotubes and 2D Periodic Arrays Self-Assembled from DNA Multi-helix Bundles. J. Am. Chem. Soc. 2012, 134 (3), 1606-1616.

(39) Woods, D.; Doty, D.; Myhrvold, C.; Hui, J.; Zhou, F.; Yin, P.; Winfree, E., Diverse and Robust Molecular Algorithms Using Reprogrammable DNA Self-Assembly. Nature 2019, 567 (7748), 366-372.

(40) Dresselhaus, M. S.; Dresselhaus, G.; Saito, R., Physics of Carbon Nanotubes. Carbon 1995, 33 (7), 883-891.

(41) Saito, R.; Fujita, M.; Dresselhaus, G.; Dresselhaus, M. S., Electronic Structure of Chiral Graphene Tubules. Appl. Phys. Lett. 1992, 60 (18), 2204-2206.

(42) Barish, R. D.; Schulman, R.; Rothemund, P. W. K.; Winfree, E., An Information-Bearing Seed for Nucleating Algorithmic SelfAssembly. Proc. Natl. Acad. Sci. U.S.A. 2009, 106 (15), 6054-6059.

(43) Mohammed, A. M.; Schulman, R., Directing Self-Assembly of DNA Nanotubes Using Programmable Seeds. Nano Lett. 2013, 13 (9), 4006-4013.

(44) Jorgenson, T. D.; Mohammed, A. M.; Agrawal, D. K.; Schulman, R., Self-Assembly of Hierarchical DNA Nanotube Architectures with Well-Defined Geometries. ACS Nano 2017, 11 (2), 1927-1936.

(45) Minev, D.; Wintersinger, C. M.; Ershova, A.; Shih, W. M., Robust Nucleation Control via Crisscross Polymerization of Highly Coordinated DNA Slats. Nat. Commun. 2021, 12 (1), 1741.

(46) Reishus, D.; Shaw, B.; Brun, Y.; Chelyapov, N.; Adleman, L., Self-Assembly of DNA Double-Double Crossover Complexes into High-Density, Doubly Connected, Planar Structures. J. Am. Chem. Soc. 2005, 127 (50), 17590-17591.

(47) Lee, J. Y.; Kim, Y.-J.; Lee, C.; Lee, J. G.; Yagyu, H.; Tabata, O.; Kim, D.-N., Investigating the Sequence-Dependent Mechanical Properties of DNA Nicks for Applications in Twisted DNA Nanostructure Design. Nucleic Acids Res. 2018, 47 (1), $93-102$.

(48) Jung, W. H.; Chen, E.; Veneziano, R.; Gaitanaros, S.; Chen, Y., Stretching DNA Origami: Effect of Nicks and Holliday Junctions on the Axial Stiffness. Nucleic Acids Res. 2020, 48 (21), 12407-12414. 


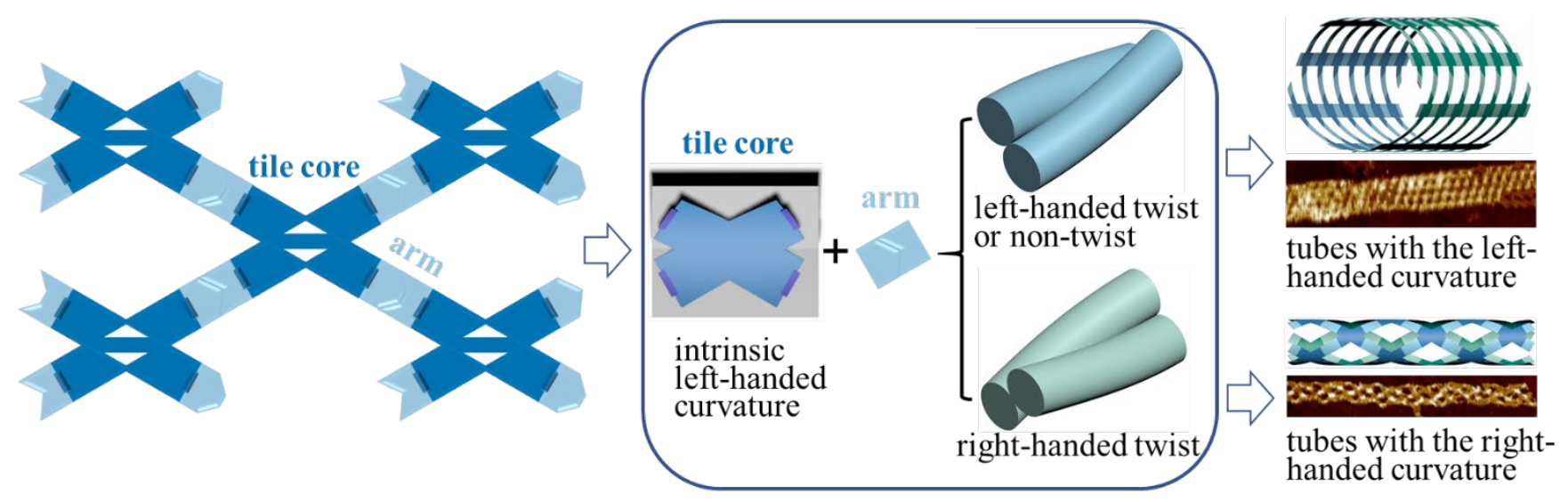

